\title{
Archéopages
}

Archéopages

Archéologie et société

40 | 04-07/2014

Villages

\section{La problématique des « villages ouverts »}

Jean Vaquer et Muriel Gandelin

\section{OpenEdition}

\section{Journals}

Édition électronique

URL : https://journals.openedition.org/archeopages/587

DOI : 10.4000/archeopages.587

ISSN : 2269-9872

\section{Éditeur}

INRAP - Institut national de recherches archéologiques préventives

\section{Édition imprimée}

Date de publication : 1 juin 2015

Pagination : 16-17

ISSN : 1622-8545

\section{Référence électronique}

Jean Vaquer et Muriel Gandelin, «La problématique des « villages ouverts » », Archéopages [En ligne], 40 | 04-07/2014, mis en ligne le 01 juillet 2016, consulté le 26 janvier 2022. URL : http:// journals.openedition.org/archeopages/587 ; DOI : https://doi.org/10.4000/archeopages.587 
1. « Le Pirou n, Valros, Hérault: la vue aérienne du site met en évidence un semis concentré de fosses circulaires qui apparaissent nettement sur l'encaissant de couleur claire.

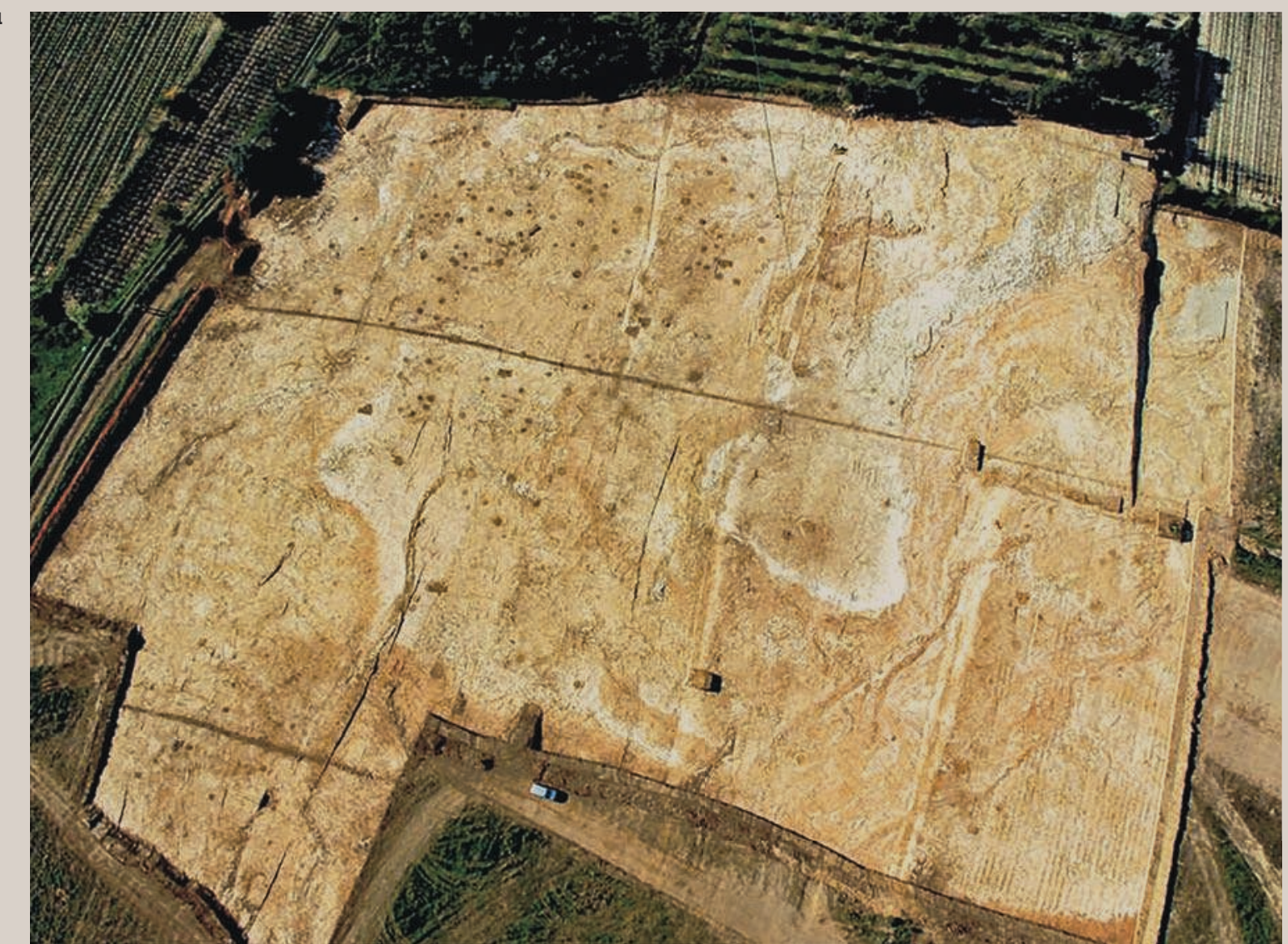

La

\section{problématique des « villages} ouverts "

\author{
Jean Vaquer Inrap, UмR 5608 «Travaux et recherches \\ archéologiques sur les cultures, les espaces et les sociétés
}

\section{Muriel Gandelin Inrap, UMR 5608 «Travaux et}

L'étude des villages ouverts néolithiques du sud de la France se heurte souvent à l'arasement général des sites qui n'ont pas conservé, pour la plupart d'entre eux, leurs niveaux de circulation et qui sont fréquemment érodés ou détruits sur plusieurs dizaines de centimètres. D'autre part, le bâti méditerranéen faisant une large part à l'utilisation de la terre crue comme matériau de construction sans ancrage au sol, il est fréquent qu'aucun témoin d'architecture ne soit détectable.

Les sites ouverts regroupant un nombre important de structures en creux et qui peuvent être interprétés comme des villages sont toutefois nombreux, notamment dans le Chasséen ancien méridional : sur l'aire de repos de Valros, au lieu dit «Le Pirou », plus de 150 fosses de stockage s'organisent au sein d'un espace limité d'environ 1 ha. À SaintAntoine, Saint-Aunès (Hérault), la même organisation, comptant plus de 250 fosses, semble correspondre à une installation villageoise d'environ $1,2 \mathrm{ha}$ fréquentée durant tout le Chasséen (Furestier et al., 2012). C'est la même configuration qui a été relevée au Crès à Béziers, Hérault (environ 1,2 ha) (Loison et al., 2004) ou aux Plots à Berriac, Aude (environ 1,6 ha) (Duday, Vaquer, 2003). Si les sites de ce type ne sont pas inhabituels, ils sont rarement connus sur la totalité de leur surface et l'absence de délimitation de l'espace ne permet pas d'appréhender clairement leurs limites. Le site du Pirou est un des seuls à être connu sur la majeure partie de son emprise. Cette occupation chasséenne, découverte à la faveur d'un décapage d'un seul tenant de $25000 \mathrm{~m}^{2}$, est installée sur le versant sud d'une petite éminence miocène [ill. 1]. Flle apparaît relativement circonscrite, et les décapages extensifs menés sur les parcelles voisines, sur une surface de plus 9 ha correspondant à l'implantation d'une aire de repos autoroutière, montrent qu'elle ne se poursuit pas vers le sud ou le nord. À l'ouest, un net dénivelé constitue une limite géographique naturelle. Directement à l'est du site, les témoins chasséens disparaissent sur près de $250 \mathrm{~m}$ avant l'apparition d'un second groupe de fosses qui pourrait correspondre à une autre occupation. Toutes les structures conservées au Pirou sont partiellement détruites, sur au moins $0,60 \mathrm{~m}$, par des phénomènes taphonomiques dont les principaux sont la mise en terrasses, la mise en culture et l'érosion. Aucun niveau de sol n'est conservé et l'ensemble des aménagements préservés sont des structures en creux. Dans ce contexte, l'absence de foyers et la grande rareté des trous de poteaux, qui pourraient témoigner d'architectures anciennes, ne peuvent être jugées 


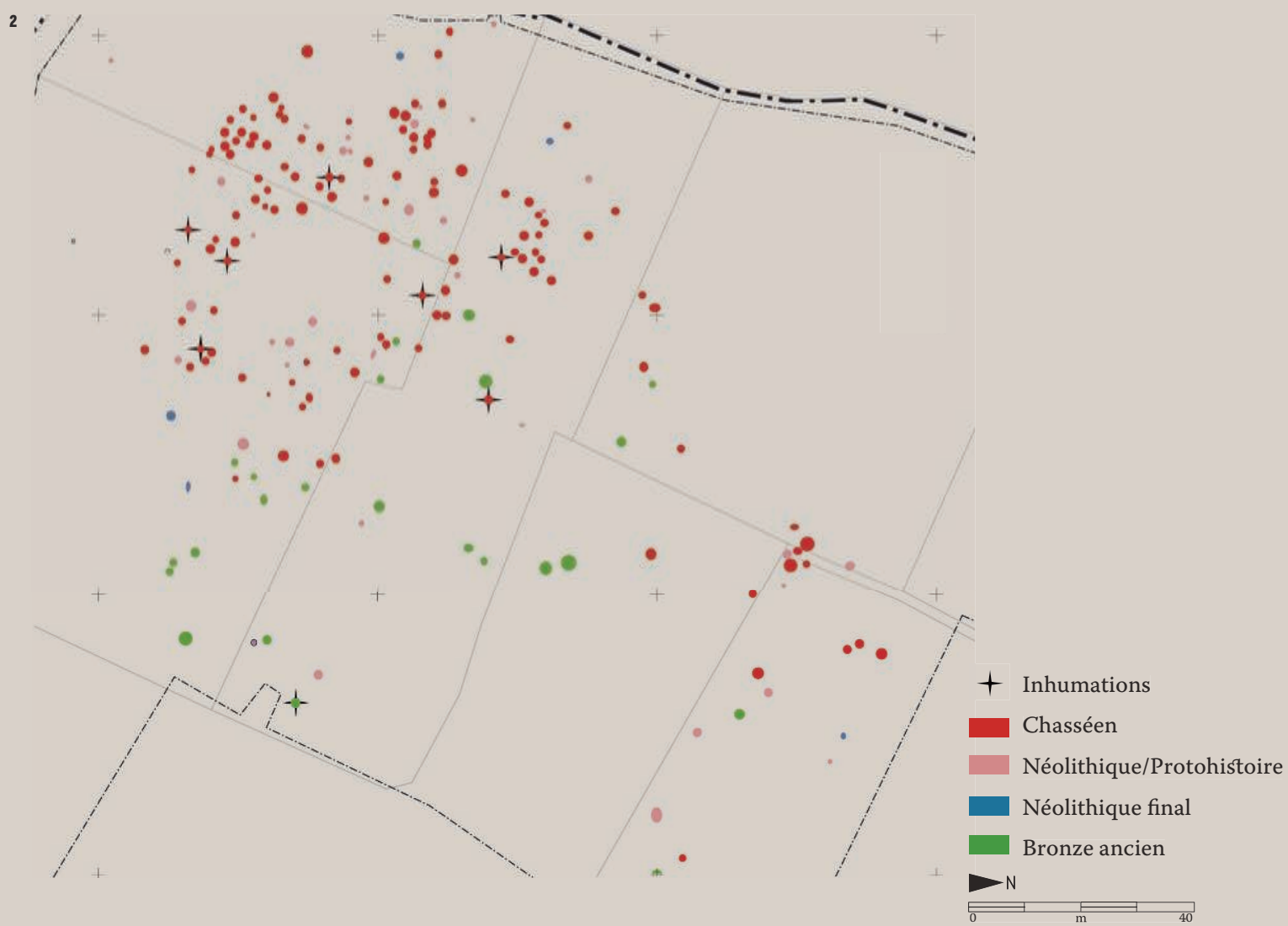

comme significatives du point de vue archéologique. De même, il n'est pas exclu que les espaces vides visibles sur le plan du site ou ceux ne contenant que des structures de l'âge du Bronze correspondent en fait à des zones où les structures chasséennes ont été totalement détruites, puisque leur profondeur est généralement inférieure à celle des structures de l'âge du Bronze [ill. 2]. Il apparaît donc nettement que, même à la faveur de décapages extensifs, l'état dans lequel on perçoit de tels sites est conditionné par la taphonomie des vestiges archéologiques. À cette première difficulté s'ajoutent les notions de durée et de rythmicité des occupations. Au Pirou, le mobilier récolté témoigne clairement du caractère domestique de cette installation du Chasséen ancien, mais aucun élément ne permet de s'assurer de la synchronie absolue des fosses fouillées. Les datations radiocarbones réalisées montrent une homogénéité qui suggère une fréquentation du site qui paraît courte, mais dont la durée réelle est impossible à fixer. Les analyses paléoenvironnementales tendent, quant à elles, à montrer que cette installation s'inscrivait dans un paysage ouvert, fortement anthropisé. L'étude malacologique autorise l'hypothèse que les populations locales pratiquaient un sylvo-pastoralisme divaguant dans un paysage constitué de prairies occupées par quelques arbres clairsemés. On voit bien que la question des modes de subsistance et notamment des pratiques agropastorales est centrale pour l'interprétation de ces sites ouverts à concentration de fosses puisque des pratiques telles que l'agriculture sur brûlis ou l'élevage extensif ont une influence directe sur les formes de l'habitat. Ainsi, les 150 fosses chasséennes du Pirou pourraient résulter de l'accrétion d'aménagements liés au stockage des semences par une vraie communauté villageoise sur une période courte de quelques années ou bien du cumul en palimpseste de ce genre de structures sur une plus longue durée par un groupe plus restreint, éventuellement familial. L'analyse anthropobiologique des défunts peut contribuer à ce débat puisque les six sujets féminins et l'enfant inhumés sur le site constituent, du point de vue de leur identité biologique, un groupe hétérogène évoquant «l'inhumation ponctuelle de défunts venant de groupes distincts " (étude en cours d'Y. Gleize, Inrap et G. Goude, CNRS). Notons toutefois qu'une pratique exogame des mariages pourrait aussi expliquer cette variabilité.

Force est de constater que

l'interprétation de tels sites n'est pas aisée et qu'elle est toujours soumise à caution : l'état de conservation des vestiges et le palimpseste des occupations constituent les deux principaux écueils auxquels se heurte l'archéologue.
Références bibliographiques

DUDAY H., VAQUER J., 2003, «Les sépultures chasséennes du site des Plots, Berriac (Aude)», in Chambon P., Leclere J. (Dir.), Les Pratiques funéraires néolithiques avant 3500 av. J.-C. en France et dans les régions limitrophes, Actes de la table ronde de Saint-Germain-en-Laye, 15-17 juin 2001, Paris, Société préhistorique française (coll. Mémoires, 33), p. 73-80.

Furestier R., Sendra B., Gourlin B., Cockin G., Gourichon L., Howarth L., LÉa V., Legrand A. LEPÈre C., Michel J., Rousselet O.

Servelle C., 2012, «Évolution du Chasséen montpelliérain : premiers résultats des fouille préventives du site de la ZAC Saint-Antoine à Saint-Aunès (Hérault) », in PerRIN T., SÉNÉPART I., Cauliez J., Thirault E., Bonnardin S. (Dir.), Dynamismes et rythmes évolutifs des sociétés de la Préhistoire récente, Actes des $9^{e}$ Rencontres méridionales de Préhistoire récente, Royan, 8-9 octobre 2010, Toulouse, Archives d'écologie préhistorique, p. 197-214.

Loison G., Fabre V., Villemeur I., 2004, Le Crès, habitats préhistoriques en bordure de l'Orb, structures domestiques et sépultures du Chasséen ancien, Rapport final d'opération, Montpellier Inrap, 304 p. 